\title{
Regulation of deactivation of photoreceptor G protein by its target enzyme and cGMP
}

\author{
Vadim Yu. Arshavsky* \& M. Deric Bownds*†
}

* Laboratory of Molecular Biology, $†$ Neuroscience Training Program, Department of Zoology, University of Wisconsin, Madison, Wisconsin 53706, USA

THE photoreceptor $G$ protein, transducin, is one of the class of heterotrimeric $\mathbf{G}$ proteins that mediates between membrane receptors and intracellular enzymes or ion channels. Light-activated rhodopsin catalyses the exchange of GDP for GTP on multiple transducin molecules. Activated transducin then stimulates cyclic GMP phosphodiesterase by releasing an inhibitory action of the phosphodiesterase $\gamma$-subunits. This leads to a decrease in cGMP levels in the rod, and closure of plasma membrane cationic channels gated by CGMP $^{1-4}$. In this and other systems, turn-off of the response requires the GTP bound to $G$ protein to be hydrolysed by an intrinsic GTPase activity ${ }^{5-7}$. Here we report that the interaction of transducin with cGMP phosphodiesterase, specifically with its $\gamma$-subunits, accelerates GTPase activity by several fold. Thus the $\gamma$-subunits of the phosphodiesterase serve a function analogous to the GTPase-activating proteins that regulate the class of small GTP-binding proteins. The acceleration can be partially suppressed by cGMP, most probably through the non-catalytic cGMP-binding sites of phosphodiesterase $\alpha$ and $\beta$-subunits. This cGMP regulation may function in light-adaptation of the photoresponse as a negative feedback that decreases the lifetime of activated cGMP phosphodiesterase as light causes decreases in cytoplasmic cGMP.

We reconstituted photoreceptor membranes which retained transducin but were depleted of cGMP phosphodiesterase (PDE), with purified PDE or its $\gamma$-subunits $\left(\mathrm{PDE}_{\gamma}\right)$. Photoreceptor membranes containing transducin tightly bound to bleached rhodopsin were obtained by illuminating the membranes in the absence of nucleotides ${ }^{8}$. More than $80 \%$ of the transducin remains bound during multiple hypotonic washes that remove more than $98 \%$ of the PDE from the membranes. This transducin will turnover if GTP is added: the $\alpha$-subunit of transducin binds GTP, dissociates from rhodopsin, and slowly hydrolyses GTP to GDP with an intrinsic GTPase activity. It is this GTPase activity that can be altered if the membranes are first permitted to rebind PDE, by preincubating them with PDE purified from toad rod outer segments to more than $98 \%$ homogeneity (Fig. 1). Because the $\gamma$-subunit of PDE is a primary site of PDE interaction with transducin ${ }^{9}$, a similar reconstitution with membranes was also done using bovine recombinant $\mathrm{PDE}_{\gamma}$ (ref. 10). To measure the rate of transducin GTPase activity we started the reaction by adding GTP in an amount less than the transducin present in the sample, so that a single turnover of GTP hydrolysis on each activated transducin molecule occurred. The binding of GTP with transducin under the conditions used here occurs at time zero and the subsequent monitoring of inorganic phosphate $\left(\mathrm{P}_{\mathrm{i}}\right)$ formation reflects the time course of a single synchronized turnover of transducin-bound GTP hydrolysis ${ }^{11,12}$. The rate constant of the GTPase reaction can be obtained from an exponential fitting of the data.

The rate of the transducin GTPase reaction can be significantly accelerated both by PDE and by $\operatorname{PDE}_{\gamma}$ (Fig. 1). The bottom curve displays the basal GTPase rate of transducin bound to the membranes $(0.034 \pm 0.003$ turnovers per second, $n=6)$. Addition of $\mathrm{PDE}$ or $\mathrm{PDE}_{\gamma}$ accelerates the rate until saturation $\left(0.143 \pm 0.02\right.$ turnovers per second, $n=4$ for $\mathrm{PDE}_{\gamma}$ and $0.14 \pm$ 0.019 turnovers per second, $n=3$ for PDE) is observed at $\sim 0.3 \mu \mathrm{M}$ PDE or $\sim 0.6 \mu \mathrm{M}$ PDE $_{\gamma}$. Because each PDE molecule contains two $\gamma$-subunits ${ }^{13}$, we conclude that the extent of
GTPase acceleration in both experiments is determined by the concentration of $\operatorname{PDE}_{\gamma}$. Figure 2 demonstrates that this acceleration of GTPase activity is slowed down by cGMP $(0.065 \pm 0.007$ turnovers per second; $n=3$ ), and that this slowing requires the presence of the $\alpha$ - and $\beta$-subunits of PDE which contain catalytic and non-catalytic cGMP-binding $\operatorname{sites}^{14-16}$. The bottom curve shows that cGMP has no effect on membrane-bound transducin in the absence of PDE. It also does not alter the accelerated GTPase rate caused by addition of $\mathrm{PDE}_{\gamma}$ (top curve). It is only if PDE is present as the $\alpha \beta \gamma_{2}$ heterotetramer that cGMP suppresses the accelerated GTPase activity. Two observations ${ }^{12}$ suggest that the cGMP-sensitivity is conferred through the non-catalytic binding sites located on the PDE $\alpha$ and $\beta$-subunits. The half-saturation of the cGMP effect on transducin GTPase in a suspension of disrupted rod outer segments is very close to the reported value of the dissociation constant $\left(K_{\mathrm{D}}\right)$ for the PDE non-catalytic cGMP-binding sites ${ }^{14}$ and $\sim 100$-fold lower than the reported Michaelis constant $\left(K_{M}\right)$ values associated with the catalytic site of amphibian PDE $^{17}$. Further, cAMP can bind to and be hydrolysed by the catalytic site, but cannot substitute for cGMP at the site that regulates GTPase.

Our findings have several implications for understanding photoreception. It has been a paradox that the rate of transducin GTPase measured in vitro ${ }^{18,19}$ is too slow to explain the relatively

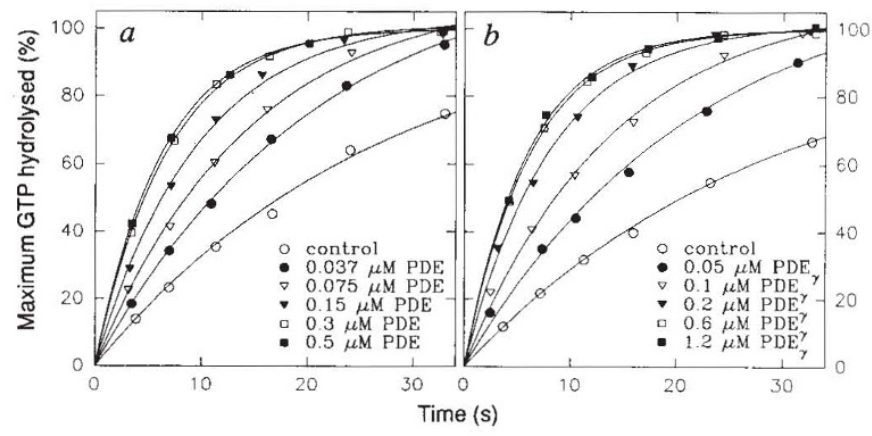

FIG. 1 Acceleration of the transducin GTPase reaction by PDE (a) and recombinant $\mathrm{PDE}_{\gamma}(b)$. Photoreceptor membranes $(20 \mu \mathrm{M}$ rhodopsin) with more than $98 \%$ of their PDE extracted but containing $\sim 1.6 \mu \mathrm{M}$ transducin were preincubated with purified PDE or recombinant $\mathrm{PDE}_{\gamma}$ at the indicated concentrations. The reaction was started by adding $0.2 \mu \mathrm{M}\left[\gamma-{ }^{32} \mathrm{P}\right] \mathrm{GTP}$ and terminated by perchloric acid. The time course of $\gamma{ }_{-}{ }^{32} \mathrm{P}_{\mathrm{i}}$ liberation by the GTPase reaction is shown; in all curves $100 \%$ hydrolysis corresponds to complete hydrolysis of the $0.2 \mu \mathrm{M}$ GTP added. The data for test membranes alone and for the highest concentrations of PDE or $\mathrm{PDE}_{\gamma}$ are approximated by single exponents. More complicated kinetics are observed for nonsaturating concentrations of $\mathrm{PDE}$ and $\mathrm{PDE}_{\gamma}$ and the corresponding lines are hand drawn. The data are taken from one of three $(a)$ or four $(b)$ similar experiments.

METHODS. The isolation of toad rod outer segments and measurements of single-turnover GTPase activity of transducin was as described in ref. 12. All experiments were done in buffer $\mathrm{A}$, which contains $100 \mathrm{mM}$ potassium isethionate $10 \mathrm{mM}$ sodium isethionate, $5 \mathrm{mM} \mathrm{MgCl}_{2}, 1 \mathrm{mM}$ dithiothreitol and $15 \mathrm{mM}$ HEPES, $\mathrm{pH} 7.8$. Test membranes retaining $>80 \%$ of their transducin and depleted by $>98 \%$ of their PDE were obtained by washing bleached rod outer segments twice with buffer $A$ and twice with buffer B: $5 \mathrm{mM}$ Tris- $\mathrm{HCl}, \mathrm{pH} 8.0,0.5 \mathrm{mM}$ EDTA and $1 \mathrm{mM}$ dithiothreitol. To prepare purified $\mathrm{PDE}$, non-bleached rod outer segments were washed twice with buffer $\mathrm{A}$ and three times with $5 \mathrm{mM}$ Tris- $\mathrm{HCl}, \mathrm{pH} 8.0$, containing $5 \mathrm{mM} \mathrm{MgCl}$ and $1 \mathrm{mM}$ dithiothreitol. Membranes were then bleached to achieve tight binding of most transducin with rhodopsin, and PDE was extracted by buffer B. After concentrating the extract with a Centricon-30 cartridge (Amicon), PDE was purified by a gel-filtration on a Superose- 6 column (Pharmacia) equilibrated with buffer B containing $10 \mathrm{mM} \mathrm{NaCl}$. The only detectable impurity in the PDE fraction was $\sim 1-2 \%$ of transducin's $\alpha$-subunit which was removed by subsequent chromatography on a protein-A-Superose column (Pharmacia) with immobilized anti-transducin antibodies $4 \mathrm{~A}$ (ref. 30). SDS-polyacrylamide gel electrophoresis of this preparation showed three bands of 92,87 and $14 \mathrm{~K}$ corresponding to PDE $\alpha-\beta$ - and $\gamma$-subunits. Purity was $>98 \%$. 


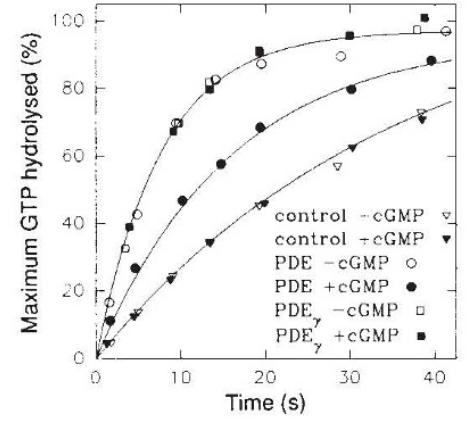

FIG. 2. CGMP reverses transducin GTPase acceleration by PDE but not PDE Single-turnover GTPase measurements were done as described in the legend to Fig. 1. Subsaturating concentrations of PDE $(0.2 \mu \mathrm{M})$ and $\mathrm{PDE}_{\gamma}(0.4 \mu \mathrm{M})$ which caused the same extent of GTPase acceleration were used. cGMP was added at a saturating concentration of $100 \mu \mathrm{M}$ where indicated. To prevent cGMP hydrolysis by PDE during the experiment the PDE inhibitor Zaprinast (100 $\mu \mathrm{M}$; May \& Baker) was added to all samples. Zaprinast itself did not influence the GTPase reaction (not shown). All the data are taken from one of three similar experiments and approximated by single exponents revealing GTPase rates of $0.031 \mathrm{~s}^{-1}$ for test membranes alone, $0.063 \mathrm{~s}^{-1}$ for the membranes in the presence of both PDE and CGMP and $0.13 \mathrm{~s}^{-1}$ for all other conditions.

fast termination of PDE activation observed under similar conditions ${ }^{20}$. More recent work indicates that transducin GTPase can be faster under more physiologial conditions ${ }^{11,21-23}$, but the mechanism of GTPase acceleration has remained unclear. The data of Fig. 1 show that PDE itself serves as a GTPase-activating factor. The maximal GTPase rate observed in this reconstitution study $\left(\sim 0.15 \mathrm{~s}^{-1}\right)$ is still about 10 -fold slower than the rate of the recovery from a photoresponse. But a more rapid rate $\left(>0.6 \mathrm{~s}^{-1}\right)$ is observed in suspensions of disrupted rod outer segments ${ }^{12}$ for the fast component of GTPase suppressed by micromolar concentrations of cGMP. Our study allows us to conclude that this faster GTPase is a property of that transducin which activates PDE, and thus the extent of PDE-dependent GTPase acceleration is higher in rod outer segment suspensions than in reconstituted membranes. More recent data (V.Y.A. et al., manuscript in preparation) shows that further concentration of rod outer segment suspensions $(>100 \mu \mathrm{M}$ rhodopsin) increases GTPase rates by at least twofold, close to the turn-off time of the photoresponse.

The data shown in Fig. 2 indicate a feedback mechanism in retinal rods based on cGMP-dependent regulation of the lifetime of activated PDE. Such a mechanism might function during rod background adaptation, when the duration and light sensitivity of the photoresponse is diminished ${ }^{24,25}$. A reasonable model is that background light depletes intracellular cGMP levels, causing dissociation of cGMP from the non-catalytic binding sites on PDE. This would accelerate the GTPase activity that terminates each PDE activation event, leading to a faster and/or smaller photoresponse. Such a mechanism might work in parallel with the known calcium feedback regulation of adaptation ${ }^{4}$.

The regulation of GTP-binding protein GTPase activity by an effector described here, although not previously described for a heterotrimeric $G$ protein, has been documented extensively for several other classes of GTP-binding proteins (for example ref. 26). It is observed for elongation and initiation factors whose GTPase activity is enhanced by ribosomes. The class of small GTP-binding proteins including the product of proto-oncogene ras interact with GTPase-activating proteins (GAPs) that may also be their effectors ${ }^{27}$. The intrinsic GTPase of the heterotrimeric signal-transducing $G$ proteins is considerably more rapid than that of the small GTP-binding proteins (for example refs 5-7), but still in several systems such as photoreception $^{2,3}$, olfaction ${ }^{28}$ and muscarinic receptor-induced potassium channel regulation ${ }^{29}$ it has seemed to be too slow to explain the rapid on-off cycle of the relevant effectors. Because acceleration has now been associated with the effector enzyme in the photoreceptor, it is relevant to search for similar mechanisms in other systems using heterotrimeric $\mathrm{G}$ proteins.

\footnotetext{
Received 4 February; accepted 8 April 1992

1. Stryer, L. A. Rev. Neurosci. 9, 87-119 (1986).

2. Liebman, P. A., Parker, K. R. \& Dratz, E. A. A. Rev, Physiol. 49, 765-791 (1987).

3. Chabre, M. \& Deterre, P. Eur. J. Biochem. 179, 255-266 (1989).

4. Chabre, M. \& Deterre, P. Eur. J. Blochem. 179, 255-266 (1989).

5. Taylor, C. W. Biochem. J. 272, 1-13(1990).

6. lyengar, R. \& Birnbaumer, L. (eds) G Proteins (Academic, San Diego, 1990).

7. Simon, M. I., Strathmann, M. P. \& Gautam, N. Science 252, 802-808 (1991)

8. Kuhn, H. Nature $\mathbf{2 8 3}, 587-589$ (1980)

9. Hurley, J. B. \& Stryer. L. J. biol. Chem. 257, 11094-11099 (1982).

10. Brown, R. L. \& Stryer, L. Proc. natn. Acad. Sci. U.S.A. 86, 4922-4926 (1989).

11. Arshavsky $\vee$ Yu Antoch, M.P. Lukjanov, K. A. \& Philippov P. P FEBS Lett 250, 353-356 (1989)

12. Arshavsky, V. Yu., Gray-Keller, M. P. \& Bownds, M. D. J. biol. Chem. 266, 18530-18537 (1991)

13. Deterre, P., Bigay, J., Forquet, F., Robert, M. \& Chabre, M. Proc. natn. Acad. Sci. U.S. A. 85, 2424-2428 (1988).

14. Yamazaki, A., Sen, I., Bitensky, M., Casnellie, J. E. \& Greengard, P. J. biol. Chem. 255, 11619-11624 (1980).

15. Charbonneau, H. et al. Proc. natn. Acad. Sci. U.S.A. 87, 288-292 (1990).

16. Li, T., Volpp. K. \& Applebury, M. L. Proc. natn. Acad. Sci. U.S. A. 87, 293-297 (1990)

17. Robinson. P. R. Kawamura, S., Abramson, B. \& Bownds. M. D. J. gen. Physiol. 76, 631-645 (1980).

18. Fung, B. K.-K., Hurley, J. B. \& Stryer, L. Proc. natn. Acad. Sci. U.S. A. 78, 152-156 (1981)

19. Baehr, W., Morita, E., Swanson, R. \& Applebury, M. L. J. biol. Chem. 257, 6452-6460 (1982).

20. Sitaramayya, A. \& Liebman, P. A. J. biol. Chem. 258, 12106-12109 (1983).

21. Dratz, E. A., Lewis, J. W., Schaechter, L. E., Parker, K. R. \& Kliger, D. S. Biochem. biophys. Res. Commun. 146, 379-386 (1987).

22. Wagner, R. Ryba, N. \& Uhl, R. FEBS Lett. 234, 44-48 (1988)

23. Vuong T. M. \& Chabre M. Proc natn. Acad. Sci. U.S.A. 88, 9813-9817 (1991)

24. Fain, G. L. J. Physiol. 261, 71-101 (1976).

24. Fain, G. L. J. Physiol. 261, 71-101 (1976).
25. Baylor, D. A., Lamb, T. D. \& Yau, K.-W. J. Physiol, 288, 589-611 (1979).

26. Bourne, H. R., Sanders, D. A. \& McCormick, F. Nature 349, 117-127 (1991).

27. Hall, A. Cell 61, 921-923 (1990).

28. Breer, H., Boekhoff, I. \& Tareilus, E. Nature 345, 65-68 (1990).

29. Breitwieser, G. E. \& Szabo, G. J. gen. Physiol. 91, 469-493 (1988)

30. Witt, P. L., Hamm, H. E. \& Bownds, M. D. J gen. Physiol \&4, 251-263 (1984).
}

ACKNOWLEDGEMENTS. We thank M. P. Gray-Keller for participating in the preliminary stage of this work, P. D. Calvert and A. Yamazaki for discussion, T. F. J. Martin and A. E. Ruoho for reading the manuscript, C. L. Dumke, M. Vidal and O. Gaide for technical support, L. Stryer and R. L. Brown for recombinant PDE, and H. E. Hamm for antibodies 4A. This work was supported by the NIH.

\section{Aminopeptidase $\mathbf{N}$ is a major receptor for the entero- pathogenic coronavirus TGEV}

\author{
Bernard Delmas*, Jacqueline Gelfi*, René L'Haridon*, \\ Lotte Katrine Vogel†, Hans Sjöström†, Ove Norén† \\ \& Hubert Laude* ${ }^{*}$
}

* Unité de Virologie et Immunologie Moléculaires, INRA,

Domaine de Vilvert, 78350 Jouy-en-josas, France

† Department of Biochemistry C, Panum Institute, DK 2200 Copenhagen N, Denmark

CORONAVIRUSES, like many animal viruses, are characterized by a restricted host range and tissue tropism'. Transmissible gastroenteritis virus (TGEV), a major pathogen causing a fatal diarrhoea in newborn pig, replicates selectively in the differentiated enterocytes covering the villi of the small intestin $e^{2}$. To investigate the molecular determinants of the infection, we characterized the surface molecule used by the virus for binding and entry into host cells. Here we report that aminopeptidase $N$, an ectoenzyme abundantly expressed at the apical membrane of the enterocytes, serves as a receptor for TGEV. Monoclonal antibodies were selected for their ability to block infection by TGEV of porcine cell lines. They recognized a brush-border membrane protein of $M_{\mathrm{r}} 150 \mathrm{~K}$, which was identified as aminopeptidase $\mathbf{N}$ by amino-terminal sequencing. Two lines of evidence supported the view that the peptidase itself

¥To whom correspondence should be addressed. 